\title{
ANÁLISE DE INDICADORES EPIDEMIOLÓGICOS DA COINFECÇÃO TUBERCULOSE-HIV
}

\author{
ANALYSIS OF EPIDEMIOLOGICAL INDICATORS OF \\ TUBERCULOSIS-HIV COINFECTION
}

Pedro Victor Farias do Nascimento Aguinaldo José de Araújo Jéssica Lins de Oliveira

Aline de Paula Rêgo Graciano Luz

Bueno Callou Bernardo de Oliveira

Tânia Maria Ribeiro Monteiro de Figueiredo

RESUMO: Objetivo: Avaliar os indicadores de cura e de abandono dos casos de tuberculose e de coinfecção tuberculose/HIV. Métodos: Estudo transversal, descritivo e quantitativo, realizado no estado da Paraíba-Brasil. A população estudada foi composta por dois grupos notificados no Sistema de Informação de Agravos de Notificação Compulsória da Secretaria de Saúde do Estado da Paraíba, durante 2013 a 2015, sendo eles: todos os casos de tuberculose e todos os casos de coinfecção tuberculose-HIV, em que ambos obtiveram cura ou abandono como situação de encerramento do tratamento. As frequências absolutas e relativas foram descritas, bem como, aplicou-se o teste de Odds Ratio. Resultados: Em relação aos doentes de tuberculose, $61 \%$ obtiveram cura e 13\% abandonaram o tratamento. Quanto aos coinfectados tuberculose/HIV, 42\% alcançaram a cura e $24 \%$ abandonaram o tratamento. Em ambos os grupos, o percentual de abandono foi maior para o tratamento autoadministrado em comparação com os que realizaram o Tratamento Diretamente Observado. Segundo teste de Odds Ratio, os sujeitos coinfectados apresentaram 2,6 mais chance de abandonar o tratamento da tuberculose comparados aos casos de tuberculose sem confirmação para o HIV. Considerações finais: $O$ abandono do tratamento da tuberculose constitui $O$ principal desafio dos órgãos de controle da doença, a adesão à terapêutica deve ser considerada como um fator imprescindível para a obtenção da cura. Portanto, os gestores e profissionais de saúde devem está atentos aos grupos populacionais em situação de maior vulnerabilidade, tanto para o adoecimento, quanto para a não adesão ao tratamento.

Palavras chave: Tuberculose. HIV. Coinfecção. Saúde Pública. 
ABSTRACT: Objective: To evaluate the indicators of cure and abandonment of cases of tuberculosis and tuberculosis / HIV co-infection. Methods: Cross-sectional, descriptive and quantitative study, conducted in the state of Paraiba-Brazil. The studied population consisted of two groups notified in the Compulsory Notification Diseases Information System of the Paraiba State Health Department, from 2013 to 2015, namely: all cases of tuberculosis and all cases of tuberculosis-HIV co-infection, in which both obtained cure or abandonment as a situation of termination of treatment. The absolute and relative frequencies were obtained, as well as, the Odds Ratio test was applied. Results: For tuberculosis patients, $61 \%$ were cured and $13 \%$ abandoned treatment. As for co-infected tuberculosis / HIV, 42\% achieved cure and $24 \%$ abandoned treatment. In both groups, the percentage of abandonment was higher in the self- administered treatment compared to those who underwent Directly Observed Treatment. According to the Odds Ratio test, that coinfected subjects presented 2.6 more chance of abandoning treatment of tuberculosis than achieving cure, compared to cases of tuberculosis without confirmation for HIV. Conclusion: The abandonment of tuberculosis treatment is the main challenge of the control organs of the disease, adherence to therapy should be considered as an essential factor to obtain cure. Therefore, managers and health professionals should pay attention to the population groups in situations of greater vulnerability, both for illness and for non-adherence to treatment.

Keywords: Tuberculosis. HIV. Coinfection. Public Healt. 


\section{INTRODUÇÃO}

A ocorrência da tuberculose (TB) transcende aspectos clínicos e biológicos, e está intrinsicamente ligada a condições sociais desfavoráveis e ao acesso limitado aos serviços de saúde. Apesar de contar com mecanismos de prevenção e tratamento passíveis de cura, a magnitude de sua morbidade e mortalidade ainda é preocupante, principalmente quando associada ao HIVIAIDS. De acordo com a Organização Mundial da Saúde (OMS), em 2018, 10 milhões de pessoas adoeceram por TB no mundo, e destas, aproximadamente 1,2 milhões chegaram a óbito, incluindo 251 mil pessoas vivendo com HIVIAIDS (PVHA). Estima-se que cerca de $8,4 \%$ de todos os casos de TB são de soropositivos (LUNA, et al. 2015; WHO, 2019).

As PVHA são até 28 vezes mais vulneráveis a adoecer por TB em comparação com a população geral, constituindo assim um grande desafio para o controle epidemiológico da TB. Por esse fato a testagem para o HIV é uma recomendação do Ministério da Saúde para todos os doentes com TB, em contrapartida, no Brasil, em 2018, 24,5\% dos casos de TB diagnosticados não realizaram a testagem anti-HIV, fato preocupante, pois trata-se de uma importante estratégia para identificação oportuna da coinfecção e introdução precoce da terapia antirretroviral (TARV), contribuindo para um melhor prognóstico do tratamento da TB (BRASIL, 2019a; WHO, 2017).

No estado da Paraíba, foram diagnosticados 1.112 casos novos de TB em 2018, com incidência e mortalidade de respectivamente, 27,5/100 mil habitantes e 1,5/100 mil habitantes. No que se refere a coinfecção TB-HIV foram notificados 116 casos no estado em 2017, equivalente a $10,8 \%$ do total de coinfecção nesse mesmo ano. Outrossim, 31,6\% dos casos de TB notificados em 2018 não realizaram o teste rápido de HIV e apenas $36,8 \%$ dos casos novos positivos iniciaram a TARV durante o tratamento da TB, fato preocupante, visto que a introdução oportuna de terapia 
para ambas infecções minimizam a ocorrência de desfechos desfavoráveis, como óbito e abandono de tratamento (BRASIL, 2019a, 2019b).

Visando o fim da TB como problema de saúde pública até o ano de 2035, a OMS lançou a estratégia global para o enfrentamento da doença, trata-se da Estratégia Pelo Fim da Tuberculose, a qual tem como objetivo reduzir o coeficiente de incidência para menos de 10 casos por 100 mil habitantes e reduzir o número de óbitos por TB em 95\% em relação ao ano de 2015 (WHO, 2016).

Diante disso, o controle da coinfecção TB/HIV é imprescindível para o alcance das metas estabelecidas pela OMS. Ademais, a não adesão e abandono do tratamento de ambas as doenças constitui um grande desafio para os órgãos de controle da TB. Visto que, a adesão é um processo que não depende exclusivamente do doente, mas de aspectos demográficos, sociais, econômicos e de acesso aos serviços de saúde, sendo necessária uma maior atenção dos gestores e profissionais de saúde aos grupos vivendo em condições de maior vulnerabilidade (LUNA, et al. 2015; SILVA, et al. 2017).

Estratégias como o Tratamento Diretamente Observado (TDO), onde o doente é acompanhado rotineiramente por um profissional de saúde, proporciona uma maior adesão ao tratamento pelo doente, entretanto, apesar de ser recomendado pelo Ministério da Saúde a todos os doentes de TB, nem todos tem acesso, o que favorece o aumento do índice de abandono do tratamento e dos óbitos causados em decorrência da coinfecção TB/HIV, assim como aumento da incidência de casos de TB e resistência aos tuberculostáticos (ARAKAWA, et al. 2017; BRASIL, 2017; LAVÔR, et al. 2016).

Nesse contexto, o presente estudo objetivou avaliar os indicadores de cura e abandono dos casos de tuberculose e dos casos de coinfecção tuberculose-HIV, visando o fornecimento de subsídios científicos aos gestores e profissionais de saúde para a otimização das ações voltadas a adesão ao tratamento da TB e consequentemente, o alcance das metas pós-2015 estabelecidas pela OMS. 


\section{MÉTODOS}

Estudo transversal descritivo com abordagem quantitativa, realizado no estado da Paraíba, localizado na região leste do Nordeste brasileiro. O estado abrange uma área de $56.467,239 \mathrm{~km}^{2}$ com população estimada de 4.018.127 milhões de habitantes distribuídos em 223 municípios, sendo o $13^{\circ}$ estado mais populoso do país (IBGE, 2019). Em relação à situação epidemiológica da tuberculose, a Paraíba está entre os estados considerados prioritários pelo Ministério da Saúde para o controle da doença no Brasil (BRASIL, 2017).

A população do estudo foi composta por dois grupos: o primeiro, todos os casos de tuberculose que obtiveram cura ou abandono como situação de encerramento do tratamento da tuberculose, e com os seguintes resultados para a realização do teste Anti-HIV: não reagente, teste em andamento e os em brancos, computados no sistema de informação. O segundo grupo, todos os casos de tuberculose configurados como coinfecção TB-HIV, após o resultado "Reagente" do teste Anti-HIV, e que obtiveram cura ou abandono como situação de encerramento do tratamento da tuberculose. Todos os casos foram notificados no Sistema de Informação de Agravos de Notificação Compulsória (SINAN) da Secretaria de Saúde do Estado da Paraíba, no período de 2013 a 2015.

Os dados foram coletados no banco de dados do SINAN-PB, no período de junho a julho de 2017, através do aplicativo Tabwin 32 versão 4.8, e posteriormente transferidos para planilhas eletrônicas do software Microsoft Excel 2010. A análise quantitativa compreendeu os resultados descritivos, considerando os valores das distribuições de frequências absolutas e relativas, foram comparadas as taxas de cura e abandono através de gráficos de tendência e em função da variável sexo e modalidade de tratamento, bem como se aplicou o teste de Odds Ratio associando os coinfectados TB-HIV e os casos de TB, em função dos indicadores cura e abandono.

O estudo é oriundo do projeto de pesquisa intitulado "Avaliação da situação epidemiológica da coinfecção tuberculose-HIV no estado da Paraíba" aprovado pelo 
Comitê de Ética em Pesquisa da Universidade Estadual da Paraíba-CEP/UEPB, com o seguinte número do parecer: 59349316.8.0000.5187, de acordo com as orientações inerentes ao protocolo de pesquisa contido na Resolução $n^{\circ}$ 466/12 do Conselho Nacional de Saúde.

\section{RESULTADOS}

No período de 2013 a 2015 foram notificados 4359 casos de TB no SINANPB, desses, 398 sujeitos também eram infectados pelo HIV. Em relação aos doentes de TB, $61 \%$ dos casos ( $n$ : 2259) obtiveram a cura e 13\% dos casos ( $\mathrm{n}$ : 500$)$ abandonaram o tratamento. Quanto aos doentes de TB coinfectados com o HIV, $42 \%$ dos casos ( $n$ : 159) alcançaram a cura da TB e 24\% (n: 94) abandonaram o tratamento da TB. Como mostra o gráfico 1:

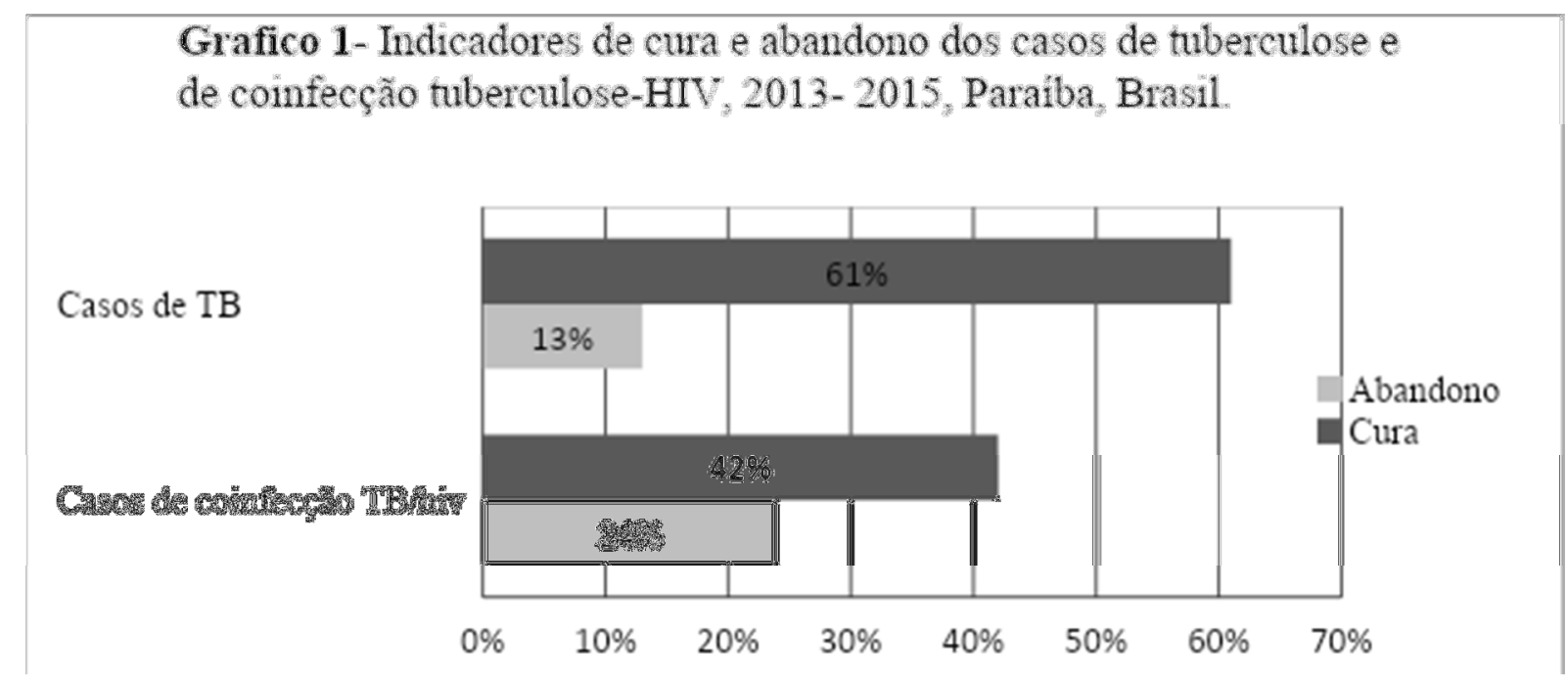

Fonte: Dados obtidos do banco de dados do Sistema de Agravos de Notificação da Secretaria Estadual de Saúde da Paraíba (SINAN-PB), 2013-2015.

Na tabela 1, estão distribuídos os casos de coinfecção TB-HIV e os casos de TB sem confirmação de infecção pelo HIV. Em relação aos coinfectados, 94 doentes abandonaram o tratamento da TB e 159 alcançaram a cura da TB. No que se refere aos casos de TB não coinfectados, 500 sujeitos abandonaram o tratamento da TB e 
2259 obtiveram a cura. Ao aplicar o teste de Odds Ratio (OR), observou-se que os sujeitos com coinfecção TB-HIV, no período estudado, apresentaram 2,6 mais chance de abandonar o tratamento da TB do que alcançar a cura, comparando-os aos doentes de TB.

Tabela 1. Casos de tuberculose e de coinfecção tuberculose-HIV em função dos indicadores da tuberculose: cura e abandono, Paraíba, Brasil, 2013-2015.

\begin{tabular}{|l|c|c|}
\hline DOENTES DE TUBERCULOSE & $\begin{array}{c}\text { Abandono do tratamento } \\
\text { da tuberculose }\end{array}$ & Cura da tuberculose \\
\hline Casos de coinfecção TB/HIV & 94 & 159 \\
\hline Casos de TB & 500 & 2259 \\
\hline
\end{tabular}

Fonte: Dados obtidos do banco de dados do Sistema de Agravos de Notificação da Secretaria Estadual de Saúde da Paraíba (SINAN-PB), 2013-2015.

$O R=(94 / 500) /(159 / 2259)=\mathbf{2 , 6}$.

O gráfico 2, mostra a tendência temporal dos indicadores de cura da TB e abandono do tratamento da TB nos casos de TB, onde observa-se uma média de $63 \%$ de cura por ano, e uma tendência decrescente para as taxas de abandono, com 17\% em 2013, 13\% em 2014 e 10\% em 2015. Vale ressaltar que, por ano, uma média de $10,7 \%$ dos casos estavam com a situação de encerramento do tratamento da TB ignorado ou em branco no banco de dados do SINAN-PB, no período da coleta de dados.

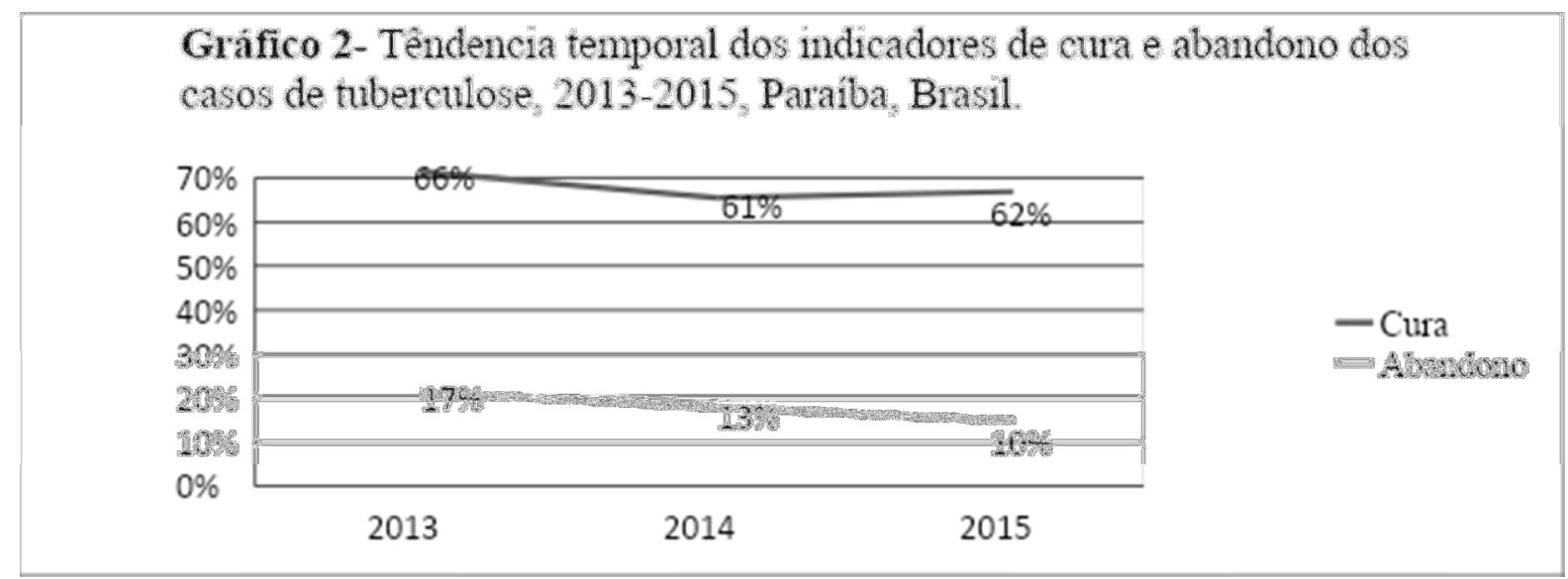

Fonte: Dados obtidos do banco de dados do Sistema de Agravos de Notificação da Secretaria Estadual de Saúde da Paraíba (SINAN-PB), 2013-2015. 
O gráfico 3 , demonstra a tendência temporal dos indicadores de cura e abandono nos casos de coinfecção TB-HIV, apresentando uma tendência constante para o indicador de cura, com média de $42 \%$ por ano. No que se refere ao indicador de abandono, destaca-se a queda significativa no percentual de casos que abandonaram o tratamento da TB, 29\% em 2013, 31,2\% em 2014 e 15,6\% em 2015.

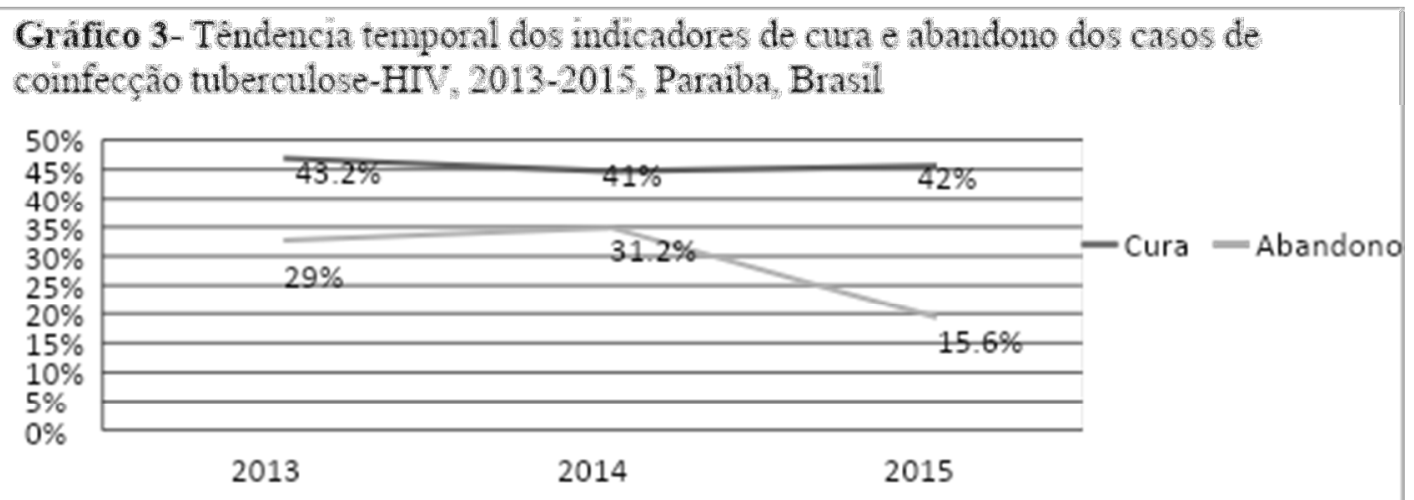

Fonte: Dados obtidos do banco de dados do Sistema de Agravos de Notificação da Secretaria Estadual de Saúde da Paraíba (SINAN-PB), 2013-2015.

Ao analisar os indicadores de cura e abandono em função do sexo, observouse que os casos de TB no sexo feminino apresentaram maior percentual de cura $(87,4 \%)$ e menor taxa de abandono do tratamento $(12,6 \%)$ em comparação com o sexo masculino (cura: $80 \%$ e abandono: $20 \%$ ). Já nos casos de coinfecção TB-HIV, ocorreu o inverso, os doentes do sexo masculino apresentaram as melhores taxas de cura (65\%) e abandono (35\%) quando comparados com o público do sexo feminino (cura: $58 \%$ e abandono: $42 \%$ ). Gráfico 4 : 


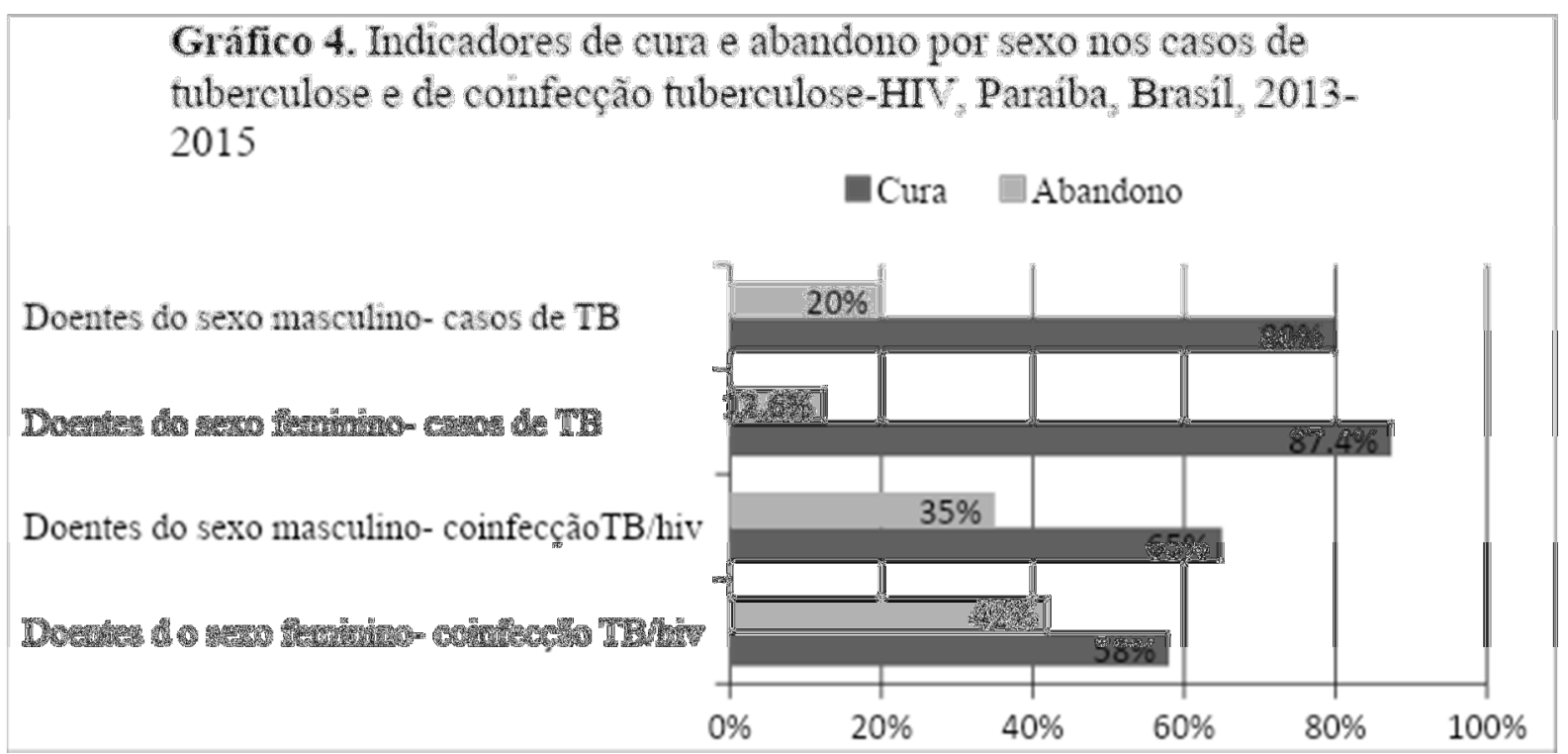

Fonte: Dados obtidos do banco de dados do Sistema de Agravos de Notificação da Secretaria Estadual de Saúde da Paraíba (SINAN-PB), 2013-2015.

No gráfico 5, pode-se observar as proporções de cura e abandono em função da modalidade de tratamento da TB (TDO e autoadministrado). Em ambos os grupos estudados o percentual de abandono foi maior para quem realiza o tratamento Autoadministrado em comparação com os que realizaram o TDO, onde as taxas de cura foram maiores e de abandono menores: casos de TB (TDO: $86,7 \%$ de cura e $12,8 \%$ abandonaram o tratamento; Autoadministrado- TDO: $79,3 \%$ de cura e $20,7 \%$ de abandono) casos de coinfecção TB-HIV (TDO: $66,2 \%$ de cura da TB e 33,8\% abandonaram o tratamento da TB; Autoadministrado: $54,7 \%$ de cura da TB e $45,2 \%$ abandonaram o tratamento da TB). 


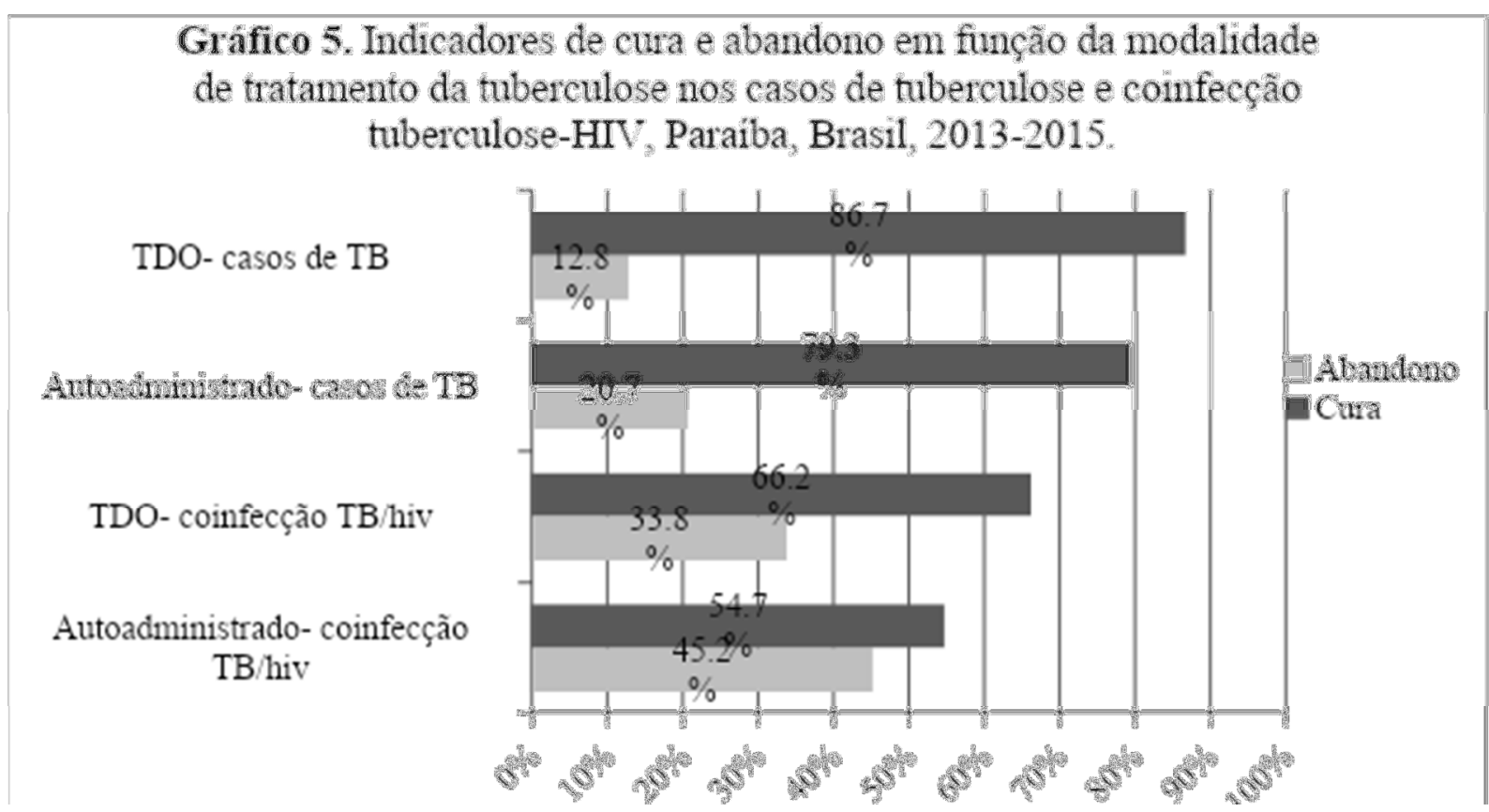

Fonte: Dados obtidos do banco de dados do Sistema de Agravos de Notificação da Secretaria Estadual de Saúde da Paraíba (SINAN-PB), 2013-2015.

\section{DISCUSSÃO}

Visando o controle da TB, o Ministério da Saúde tem estipulado metas para alcançar $85 \%$ de cura e diminuir as taxas de abandono do tratamento para $5 \%$. Entretanto, o estado da Paraíba encontra-se longe de alcançar esses patamares, sendo a situação nos casos de coinfecção TB-HIV ainda mais preocupante, pois apresenta taxas maiores de abandono e menores de cura, quando comparadas com os casos de TB sem confirmação de infecção pelo HIV (BRASIL, 2017; WHO, 2016).

Os percentuais de cura e abandono revelam a efetividade da adesão ao tratamento e das ações de controle da TB, funcionando assim, como marcadores da qualidade do processo de trabalho dos serviços de saúde (PINTO, et al. 2015). Todavia, estes percentuais podem está subestimados, visto que o banco de dados do SINAN-PB apresenta muitos casos com a situação de encerramento do tratamento da TB em branco. 
No presente estudo, observou-se que os casos de coinfecção TB-HIV apresentam uma maior chance de abandonar o tratamento da TB do que obter a cura, quando comparados com os casos não coinfectados. Sabe-se que as PVHA apresentam uma maior suscetibilidade para o adoecimento por TB pelo comprometimento do sistema imunológico em decorrência da infecção pelo HIV, bem como são mais vulneráveis para não adesão ao tratamento de ambas às doenças, sendo necessária a implementação de estratégias eficazes para o fortalecimento da adesão dos sujeitos ao tratamento (CHIRINOS, et al. 2015; FILHO, et al. 2012).

A literatura científica evidencia que a não adesão e abandono do tratamento da TB está intrinsecamente ligado aos aspectos sociais e econômicos desfavoráveis para uma boa qualidade de vida, uso de drogas e falhas relacionados ao acolhimento e atendimento nos serviços de saúde, bem como acesso dificultado ao tratamento da doença, como o diagnóstico tardio e a não implementação efetiva do TDO. Consequentemente, um alto nível de abandono, apresenta relação direta com a disseminação da doença, surgimento de bacilos multirresistentes, aumento do número de óbitos e dificuldades em alcançar as metas pactuadas pela OMS para o controle definitivo da TB (COUTO, et al. 2014; FILHO, et al. 2012; SILVA, et al. 2014).

Estudos apontam a predominância da TB no sexo masculino de faixa etária economicamente ativa, esse fato pode está associado ao estilo de vida, onde o público do sexo masculino geralmente está mais exposto a fatores de risco para o adoecimento e a um maior desinteresse na procura de serviços de saúde, em comparação com os doentes do sexo feminino. Assim, esses fatores também podem interferir na adesão ao tratamento, visto que pesquisas evidenciam que as mulheres são mais propensas a concluírem o tratamento favorecendo a obtenção da cura da TB (PINTO, et al. 2015; WHO, 2017).

O presente estudo traz um achado ao fazer comparação entre os sexos em função da TB e da coinfecção, onde observou-se que nos casos de coinfecção TBHIV os doentes do sexo masculino apresentaram as melhores taxas de cura e abandono quando comparados às mulheres. Nos casos de TB, ocorre o oposto, as mulheres foram as que apresentaram as melhores taxas. 
No tocante a modalidade de tratamento, os sujeitos dos dois grupos estudados que foram acompanhados pelo TDO obtiveram os melhores resultados para os indicadores de cura e abandono. Sabe-se que o TDO está indicado pelo Ministério da Saúde a todos os doentes de TB de forma universal; e o Serviço de Atenção Especializada (SAE) a PVHA deve articular com as Unidades Básicas de Saúde para realização do TDO compartilhado nos casos de coinfecção TB/HIV, porém, nem todos têm acesso à estratégia. (BRASIL, 2013; LAVÔR, et al. 2016).

Caso aplicado conforme o preconizado, o TDO, através do vínculo estabelecido entre os envolvidos no processo, é capaz de contribuir com o fortalecimento da adesão ao tratamento, assim como para a melhoria da qualidade de vida dos doentes, familiares e da comunidade. Contudo, vale salientar que, o TDO não pode ser resumido apenas como a administração supervisionada de medicamentos, pois fatores como vínculo, diálogo a longo prazo, medidas de educação em saúde e contato singularizado impactam diretamente em seu sucesso e consequentemente no desfecho do tratamento (REIS-SANTOS, et al., 2015). Deste modo, o profissional de saúde deve estar preparado para identificar possíveis obstáculos para a adesão ao regime terapêutico e aplicar intervenções que se encaixem e respeitem as particularidades de cada paciente.

Os doentes em tratamento autoadministrado geralmente apresentam dificuldades no seguimento do regime terapêutico, visto que o monitoramento da resposta à quimioterapia, ajuste de dose e identificação de efeitos adversos aos fármacos podem não ser feitos de modo oportuno devido ao contato reduzido com o paciente, gerando fragilidades quanto a adesão ao tratamento, o que repercute negativamente no alcance das metas estabelecidas para o controle da TB (ARAKAWA, et al. 2017; LIMA, et al. 2016; LAVÔR, et al. 2016).

Ademais, informações acerca da própria doença e necessidade da ingestão medicamentosa diária mesmo após a melhora da sintomatologia clínica requerem um contato continuado e de caráter didático, a fim de que paciente e família sintamse confortáveis para sanar dúvidas e compreendam a necessidade de prosseguir com o regime terapêutico até a finalização prevista (LIMA, et al. 2016). 


\section{CONSIDERAÇÕES FINAIS}

Os percentuais de cura e abandono tanto nos casos de TB como nos de coinfecção TB- HIV estão muito abaixo das metas preconizadas pela OMS, porém os casos de TB sem confirmação de infecção pelo HIV apresentaram os melhores resultados em relação a esses indicadores, quando comparados aos sujeitos coinfectados.

Esses indicadores podem refletir a qualidade do processo de trabalho dos serviços de saúde que acompanham esses doentes, portanto, taxas de cura e de abandono desfavoráveis podem ter uma grande contribuição da não implementação efetiva do TDO. Sabendo que o TDO é de extrema importância para o sucesso no processo de adesão, os sujeitos que realizam o tratamento autoadministrado podem apresentar uma maior vulnerabilidade para a não adesão à terapêutica, consequentemente são mais suscetíveis ao abandono do tratamento, evolução para TB multirresistente e óbito, dificultando assim no alcance das metas estabelecidas para o controle epidemiológico da doença.

No tocante à variável sexo, cabe ressaltar que os homens coinfectados apresentaram uma maior taxa de cura do que as mulheres co infectadas, divergindo dos casos de TB onde ocorreu o inverso.

Ademais, o abandono do tratamento da TB constitui o principal desafio dos órgãos de controle da doença, e a adesão à terapêutica deve ser considerado um fator imprescindível para a obtenção da cura. Portanto, para o controle efetivo da TB e da coinfecção TB-HIV, é preciso não só do comprometimento do sujeito para com a saúde, mas da família, população, gestores e profissionais de saúde a fim de diminuir as iniquidades em saúde e desigualdades sociais, que ainda são os principais contribuintes para o adoecimento por TB e não adesão ao tratamento antiTB.

Cabe ressaltar que o estudo apresentou limitações na obtenção e análise de dados, visto que o banco de dados do sistema de informação utilizado, o SINAN-PB, apresenta algumas variáveis com informações ignoradas ou em branco. Assim, se 
faz necessário a atenção dos profissionais que estão envolvidos de forma direta ou indireta na notificação dos casos no SINAN-PB.

Contudo, o presente estudo visou o fornecimento de subsídios científicos aos gestores e profissionais de saúde para otimização das políticas públicas e estratégias voltadas para adesão ao tratamento da TB, favorecendo o aumento das taxas de cura e diminuição das de abandono, requerendo atenção especial para os grupos vivendo em condições de maior vulnerabilidade, como as PVHA.

\section{REFERÊNCIAS BIBLIOGRÁFICAS}

ARAKAWA, T. et al. Programa de controle da tuberculose no contexto municipal: avaliação de desempenho. Revista de Saúde Pública. Vol. 51, n. 23, 2017.

BRASIL. Brasil livre da tuberculose: evolução dos cenários epidemiológicos e operacionais da doença. Ministério da Saúde. Secretaria de Vigilância em Saúde. Boletim Epidemiológico, Brasília: Ministério da Saúde, v. 50, n. 9, 2019a. Disponível em: https://portalarquivos2.saude.gov.br/images/pdf/2019/marco/22/2019-009.pdf. Acesso em: 20 fev. 2020.

BRASIL. Panorama epidemiológico da coinfecção TB-HIV no Brasil. Ministério da Saúde. Secretaria de Vigilância em Saúde. Boletim Epidemiológico, Brasília: Ministério da Saúde vol. 50, n. 26, p. 1-28, 2019b. Disponível em: http://www.aids.gov.br/pt-br/pub/2019/boletimepidemiologico-tb-hiv-2019. Acesso em: 20 fev. 2020.

BRASIL. Ministério da Saúde. Recomendações para o manejo da coinfecção TB/HIV em serviços de atenção especializada a pessoas vivendo com HIVIAids. Ministério da Saúde. Brasília, 2013.

COUTO, D. S. et al. Fatores determinantes para o abandono do tratamento da tuberculose: representações dos usuários de um hospital público. Revista Saúde Debate. vol. 38, n.102, p. 572-581, 2014.

CHIRINOS, N. E. C. et al. Representações sociais das pessoas com tuberculose sobre o abandono do tratamento. Revista Gaúcha de Enfermagem. vol. 36, n. esp, p. 207-214, 2015.

FILHO, M. P. S. et al. Pacientes vivendo com HIVIAIDS e coinfecção tuberculose: dificuldades associadas à adesão ou ao abandono do tratamento. Revista Gaúcha de Enfermagem. vol.33, n. 2, p. 132-145, 2012.

IBGE. Instituto Brasileiro de Geografia e Estatística. Censo 2010. Disponínel em: <https://cidades.ibge.gov.br/brasil/pb/panorama> Acesso em: 20 fev. 2020.

LAVÔR, D. C. B. S. et al. Avaliação da implantação da estratégia de tratamento diretamente observado para tuberculose em um município de grande porte. Revista da Escola de Enfermagem da USP. vol. 50, n. 2, p. 247-254, 2016.

LIMA, L.M. et al. Avaliação do acompanhamento e desfecho de casos de tuberculose em município do sul do Brasil. Revista Gaúcha de Enfermagem. vol. 37, n. 1, p. 1-7, 2016. 
LUNA, F. D. T. et al. Adherence to Tuberculosis Treatment: Programatic Vulnerability Elements, International Archives of Medicine, vol.8, p.207, 2015.

PINTO, M. L. et al. Ocorrência de casos de tuberculose em Crato, Ceará, no período de 2002 a 2011: uma análise espacial de padrões pontuais. Revista Brasileira de Epidemiologia. vol. 18, n. 2, p. 313-325, 2015.

REIS-SANTOS, B. et al. Directly observed therapy of tuberculosis in Brazil: associated determinants and impact on treatment outcome. International Journal of Tuberculosis and Lung Disease, v. 19, n. 10, p. 1188-1193, 2015.

SILVA, P. F. et al. Fatores associados ao abandono do tratamento da tuberculose pulmonar no Maranhão, Brasil, no período de 2001 a 2010. Cadernos de Saúde Pública. Vol. 30, n. 8, p. 1745-1754, 2014.

SILVA, R. D. et al. Patients' perception regarding the influence of individual and social vulnerabilities on the adherence to tuberculosis treatment: a qualitative study. BMC Public Health. vol. 725, n. 17, p. 1-9, 2017.

WORLD HEALTH ORGANIZATION. Global Tuberculosis Report. Geneva, 2019. Disponível em: https://apps.who.int/iris/bitstream/handle/10665/329368/9789241565714- eng.pdf?ua=1. Acesso em: 20 fev. 2020.

WORLD HEALTH ORGANIZATION. The end TB strategy. Geneva, 2016. 